\title{
Detection of Nonthermal X-ray structures near the Galactic Center with Chandra
}

\author{
Atsushi Senda ${ }^{1}$, Katsuji Koyama ${ }^{2}$, Ken Ebisawa ${ }^{3}$, Jun Kataoka ${ }^{4}$, and \\ Yoshiaki Sofue ${ }^{5}$ \\ ${ }^{1}$ Cosmic Radiation Laboratory, RIKEN (The Institute of Physical and Chemical Research), \\ 2-1 Hirosawa, Wako, Saitama 351-0198, Japan \\ email: senda@crab.riken.jp \\ ${ }^{2}$ Cosmic Ray Group, Department of Physics, Graduate School of Science, Kyoto University \\ ${ }^{3}$ Center for Planning and Information syntems, ISAS/JAXA \\ ${ }^{4}$ Department of physics, Faculty of Science, Tokyo Institute of Technology \\ ${ }^{5}$ Institute of Astronomy, University of Tokyo
}

\begin{abstract}
We have discovered a number of nonthermal X-ray features within central $40 \mathrm{pc}$ region of the Galactic center by analysing 600-ksec observations of Chandra archival data. Most of the detected X-ray structures exhibit small-scale knot-like morphologies and their spectra are well reproduced by single hard power-law with photon indices of 1-2. Among them, the most outstanding features are the three X-ray knots which are aligned on a straight line from the potition of Sgr A* to north-northwest direction. The X-ray properties of these knots lead us to suspect that they are X-ray jets ejected from Sgr A* in the recent past. In addition, we have obtained an indication that the summed flux of nonthermal diffuse X-rays within $30 \mathrm{pc}$ of the GC seems to be smoothly connected to the $20-100 \mathrm{keV}$ flux detected with INTEGRAL IBIS/ISGRI. These results suggest that the origin of GC hard X-rays (or High energy Gamma-rays) is not (or partly) from the Galactic nucleus.
\end{abstract}

Keywords. Galaxy: center, X-rays: ISM, ISM: jets and outflows.

\section{Introduction}

A number of nonthermal radio emissions from linear filaments has been detected around the Galactic center (GC) (e.g. Yusef-Zadeh et al. (1984), Morris (1994), LaRosa et al. (2000), LaRosa et al. (2004)). Their spatial distribution seems concentrated within only the central hundred parsecs and their spectral properties suggest that their emission mechanism should be synchrotron radiation from high energy electrons. These characteristics lead us to suspect that the GC region holds some particle accelerators. In addition, it also suggests that the magnetic field strength around the GC is extremely strong ( $B \sim 1 \mathrm{mG}$ ) inferred from the extreme linearity of these radio filaments.

Recently, discovery of nonthermal X-ray filaments has been reported with Chandra and XMM-Newton observations (Wang et al. (2002), Sakano et al. (2003), Lu et al. (2003)). Although their emission mechanism and connection to the radio filaments are still unclear. It is partly because of poor samples of X-ray filaments.

Here we present the first systematic analysis of nonthermal X-ray structures within the $17^{\prime} \times 17^{\prime}$ of the Galactic center. We also analyzed the INTEGRAL IBIS/ISGRI data and found possible relation with Chandra nonthermal diffuse X-rays. A possible interpretation of emission mechanism of diffuse hard nonthermal X-rays detected with Chandra/INTEGRAL is briefly discussed. 


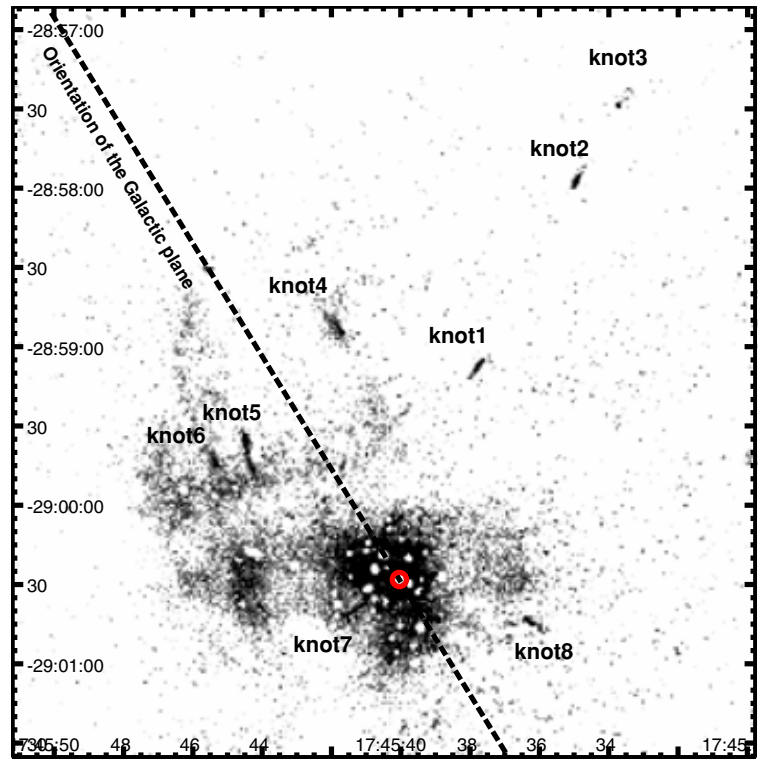

Figure 1. Continuum X-ray image of the Galactic center region with Chandra. The selected energy band is $3.42 \mathrm{keV}-6.30 \mathrm{keV}$ and the gray scale is logarithmically. Source ID of X-ray knots is also shown in the image.

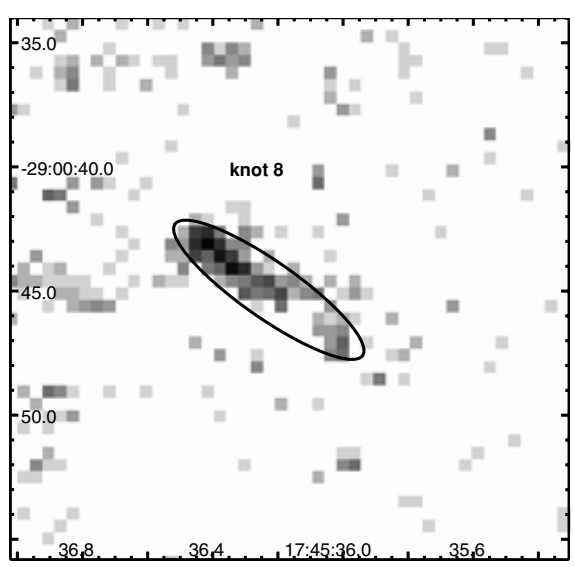

Figure 2. Close up X-ray image of knot 8 with Chandra ACIS-I. $\mathrm{X}$-ray image is constructed from 3 to 300 counts per pixel with a logarithmic scale. All of the detected point sources are excluded from the image.

\section{Analyses and Results}

\subsection{Imaging analyses}

To extract results presented in the following sections, we analyzed eleven archival Chandra ACIS-I datasets. All of these observations were performed in Timed-Exposure Faint mode except for Obs $\mathrm{ID}=242$, which was done in Timed-Exposure Very Faint mode. The total effective exposure of the combined event file amounts to $598 \mathrm{ksec}$.

To resolve diffuse nonthermal X-ray structures, we constructed continuum band image, in which there is no prominent emission line contained. From the continuum band image as is shown in Figure 1 we resolved eight prominent knot-like features, then we named them from 1 to 8 . The position of the each sources and their identifications are shown in Figure 1. A sample of Close-up X-ray image of one of the knots (knot8) is shown in Figure 2 .

\subsection{Spectral Analyses}

As a source region of each X-ray knot, we selected an elliptical region along their shape while the background spectrum is taken from the surrounding annulus with its center coordinate and width $1<r<3$ times of the radius of each source region, respectively. All of their spectra exhibit no emission lines from ions, hence we modelled each spectrum with a powerlaw model with absoption of interstellar medium taken into account. The fitting results of these knots are shown in Table 1 . The best-fit values for column density are not smaller than $N_{\mathrm{H}} \sim 1.0 \times 10^{22} \mathrm{~cm}^{-2}$, which is consistent with the value when they are located at the distance of the GC, and the possibility that these knots are foreground object is robustly rejected. These results lead us to conclude that knots $1-8$ are nonthermal X-ray sources located near the Galactic center.

We also tried to fit each spectrum with a thin-thermal plasma model (MEKAL). The model could make an acceptable fit, however, the best-fit value of an electron temperature 
was determined to be $>70 \mathrm{keV}$, which is too high a temperature to be explained by any energetic events. Therefore we thought that the application of a thermal model is unrealistic and the emission mechanism should be of nonthermal origin.

Table 1. Best-fit parameters for X-ray knot1-8 with a powerlaw model

\begin{tabular}{lcccc}
\hline Source ID & $\begin{array}{c}\text { Source counts }^{a} \\
(\text { counts })\end{array}$ & $\begin{array}{c}\text { Column Density }\left(N_{\mathrm{H}}\right) \\
\left(10^{22} \mathrm{H} \mathrm{cm}^{-2}\right)\end{array}$ & $\begin{array}{c}\text { Photon index }(\Gamma) \\
\left(N(E) \propto E^{-\Gamma}\right)\end{array}$ & $\begin{array}{c}\text { Flux }^{b} \\
\left(\mathrm{ergs} \mathrm{cm}^{-2} \mathrm{~s}^{-1}\right)\end{array}$ \\
\hline knot 1 & 501 & $8.5(4.3-14)$ & $1.3(0.3-2.2)$ & $4.1 \times 10^{-14}$ \\
knot 2 & 485 & $16(9.1-21)$ & $2.3(1.7-3.1)$ & $3.4 \times 10^{-14}$ \\
knot 3 & 295 & $10(5.3-23)$ & $1.8(0.3-2.7)$ & $3.8 \times 10^{-14}$ \\
knot 4 & 800 & $12.9(8.8-16.6)$ & $1.8(1.3-2.1)$ & $5.3 \times 10^{-14}$ \\
knot 5 & 562 & $21.4(15.6-30.2)$ & $3.2(2.2-4.6)$ & $3.7 \times 10^{-14}$ \\
knot 6 & 782 & $8.7(6.0-12.5)$ & $3.1(2.8-4.3)$ & $3.4 \times 10^{-14}$ \\
knot 7 & 183 & $1.1(<37.0)$ & $-2.4(<1.5)$ & $5.2 \times 10^{-14}$ \\
knot 8 & 419 & $10.2(7.0-18.6)$ & $0.9(-0.4-1.2)$ & $3.7 \times 10^{-14}$ \\
\hline
\end{tabular}

All the errors given in parentheses indicate the $90 \%$ confidence limits.

${ }^{a}$ Total counts from the source region after subtracting background counts. The subtracted background counts are normalized with the angular size of the region.

${ }^{b}$ An absorbed X-ray flux of $2.0-10.0 \mathrm{keV}$.

\subsection{A Possible Connection to Hard X-rays detected with INTEGRAL}

Bélanger et al. (2004) reported on the discovery of significant hard X-ray excess near the Galactic nulceus with INTEGRAL IBIS (Imager on Board the INTEGRAL Satellite) observations, which was named IGR J1745.6-2901. However, whether the excess of IGR J1745.6-2901 can be attributed to point-like source(s) or extended emission is still debatable.

For the purpose of investigating possible connection between nonthermal X-rays detected with Chandra and IGR J1745.6-2901, we constructed the 15-500 keV spectrum of IGR J1745.6-2901 from INTEGRAL IBIS/ISGRI data and then performed combined spectral fitting with 1.0-10 keV Chandra data. For the Chandra data, we use integrated spectrum of all nonthermal components detected within $17^{\prime} \times 17^{\prime}$ region with reference to the position of Sgr A*. The fitting results are shown in Table 2. At first, we fit both data sets with a powerlaw convolved with interstellar absorption. Because $N_{\mathrm{H}}$ should not be influential in the INTEGRAL data above $10 \mathrm{keV}$, the value of column density $\left(N_{\mathrm{H}}\right)$ is fixed to the best-fit value obtained from Chandra data. We cannot obtain acceptable fit with single power-law model, since the observed photon index is very different between Chandra and INTEGRAL, hence we attempt two alternative models; cutoff-powerlaw and broken-powerlaw. Both phenomenological models can successfully reproduce the 1.0 $500 \mathrm{keV}$ X-ray spectrum statistically acceptable. Both of the fits exhibit characteristic energy cutoff/break around $E_{0} \sim 25 \mathrm{keV}$.

Table 2. Best-fit parameters for nonthermal hard X-rays of the Galactic center

\begin{tabular}{lcccc}
\hline Model & $\begin{array}{c}\text { Photon index 1 } \\
\left(N(E) \propto E^{-\Gamma_{1}}\right)\end{array}$ & $\begin{array}{c}\text { Cutoff/Break energy } \\
\left(E_{0} \mathrm{keV}\right)\end{array}$ & $\begin{array}{c}\text { Photon index 2 } \\
\left(N(E) \propto E^{-\Gamma_{2}}\right)\end{array}$ & $\begin{array}{c}\text { Reduced } \chi^{2} \\
\left(\chi^{2} / \text { d.o.f. }\right)\end{array}$ \\
\hline powerlaw & $2.61(2.51-2.75)$ & - & - & $154.3 / 84$ \\
cutoff-powerlaw & $1.42(1.36-1.57)$ & $24.8(21.2-28.2)$ & - & $59.0 .3 / 83$ \\
broken-powerlaw & $1.53(1.44-1.65)$ & $25.3(22.3-28.7)$ & $3.35(3.10-3.60)$ & $53.4 / 82$ \\
\hline
\end{tabular}

All the errors given in parentheses indicate the $90 \%$ confidence limits. 
If the suprathermal electrons exist near the Galactic center, they should emit X-rays via bremsstrahlung. Energy loss rate by Coulomb interaction and bremsstrahlung are given as follows (Hayakawa(1969));

$$
\begin{gathered}
-\left(\frac{d E}{d t}\right)_{\mathrm{cou}}=7.62 \times 10^{-9} n_{\mathrm{H}}(3 \ln \gamma+18.8) \mathrm{eV} \mathrm{s}^{-1} \\
-\left(\frac{d E}{d t}\right)_{\text {bremss }}=1.37 \times 10^{-16} n_{\mathrm{H}}(\ln \gamma+0.36) E \mathrm{eV} \mathrm{s}^{-1}
\end{gathered}
$$

Since the X-ray emitting electrons have energies near MeV, energy loss of Coulomb interaction must dominate. But the cooling time of the nonthermal electrons can be expressed as follows (Rephaeli (1979));

$$
\begin{gathered}
\tau_{\text {cou }} \sim 4.3 \times 10^{4} \beta\left(\frac{n}{1 \mathrm{~cm}^{-3}}\right)^{-1}\left(\frac{E}{1 \mathrm{MeV}}\right) \mathrm{yr} \\
\tau_{\text {bremss }} \sim 4.3 \times 10^{8}\left(\frac{n}{1 \mathrm{~cm}^{-3}}\right)^{-1}\left(\frac{E}{1 \mathrm{MeV}}\right) \mathrm{yr}
\end{gathered}
$$

Thus, Coulomb interaction dominates the cooling time of suprathermal electrons in a typical condition of the Interstellar medium. By equating $\tau_{\text {cou }}=\tau_{\text {age }}$, we can obtain the break energy of the electron;

$$
E_{\text {cou }} \sim 23.2 \beta \frac{n}{1 \mathrm{~cm}^{-3}} \frac{\tau_{\text {age }}}{10^{3} \mathrm{yr}} \mathrm{keV}
$$

Masai et al. (2002) proposed that the suprathermal electrons can be generated from cooler $\left(k T_{e} \sim 0.2 \mathrm{keV}\right)$ thermal plasma via stochastic accerelation. The hard component of the Galactic diffuse X-ray emission can be explained by the emission by the electrons of "suprathermal" populations.

\section{Acknowledgements}

We express particular thanks to K. Makishima and A. Bamba for their fruitful comments. This work is financially supported by Special Postdoctoral fellowship program of RIKEN.

\section{References}

Bélanger, G., et al., 2004, A\&A, 601, L163

Ekers, R. D., van Gorkom, J. H., Schwarz, U. J., \& Goss, W. M., 1983, A\&̈A, 122, 143

Hayakawa, S., 1969, Cosmic Ray Physics, Nuclear and Astrophysical Aspects (New York: WileyInterscience)

Lang, C. C., Morris, M., \& Echevarria, L., 1999, ApJ, 526, 727

LaRosa, T. N., Kassim, N. E., Lazio, T. J. W., \& Hyman, S. D. 2000, AJ, 119, 207

LaRosa, T. N., Nord, M. E., Lazio, T. J. W., \& Kassim, N. E., 2004, ApJ, 607, 302

LaRosa, T. N., Brogan, C. L., Shore, S. N., Lazio, T. J., Kassim, N. E., \& Nord, M. E., 2005, ApJ, in press, astro-ph/0505244

Lu, F. J., Wang, Q. D., \& Lang, C., 2003, AJ, 126, 319

Masai, K., Dogiel, V. A., Inoue, H., Schönfelder, V., and Strong, A. W., 2002, ApJ, 581, 1071

Morris, M., 1994, in the Nuclei of Normal Galaxies, ed. R. Genzel \& A. I. Harris (NATO ASI Ser. C, 445; Dordrecht: Kluwer), 185

Rephaeli, Y., 1979, ApJ, 227, 364

Sakano, M., Warwick, R. S., Decourchelle, C., \& Predehl, P., 2003, MNRAS, 340, 747

Wang, Q. D., Lu, F., \& Lang, C., 2002, ApJ, 581, 1148

Yusef-Zadeh, F., Morris, M., \& Chance, D. 1984, Nature, 310, 557 


\section{Discussion}

Bosch-Ramon: Did you infer the kinetic luminosity of the ejection (when "knots" were ejected)?

SENDA: It is rather difficult because the X-ray spectra of "knots" exhibit completely nonthermal features, hence we cannot estimate the emission measure (and then temperature) of these knots from our analyses.

Pietsch: Can you explain the difference between the histogram (intensity) in the observations 2 years apart?

SENDA: I think the difference is due to statistical fluctuation because the intensity of each "knot" is very faint (Photon counts of each "knot" is typically only $300-500$ cnts/ $600 \mathrm{ksec})$. 